\title{
Environmental and safety assessments of an industrial production of levulinic acid via acid catalyzed dehydration
}

\author{
Samir I. Meramo-Hurtado ${ }^{1,}{ }^{*}$, Karina A. Ojeda², Eduardo Sanchez-Tuiran².
}

${ }^{1}$ Bussines management and productivity research group, Industrial Engineering Program, Fundación Universitaria Colombo International. Av. Pedro Heredia Sector Cuatro Vientos \#31-50. Cartagena, Colombia

${ }^{2}$ Process Design and Biomass Utilization Research Group (IDAB), Chemical Engineering Program, University of Cartagena. Campus Piedra de Bolívar. Street 30 \# 48-152. Cartagena, Colombia.

Corresponding author*: E-mail: smeramoh@unicartagena.edu.co 


\section{Table S1. Mass and Energy balance for Pretreatment Unit}

\begin{tabular}{|c|c|c|c|c|c|c|c|c|c|c|c|c|c|c|c|c|c|c|c|c|c|c|c|c|c|}
\hline Mass flow (TPE) & 2000 & 32000 & 5818 & 972 & 57790 & 32000 & 204429 & 04429 & 204429 & 91483 & 1483 & 112945 & 21570 & 91375 & 21570 & 37 & 19133 & 2474 & 54 & 19187 & 97 & 19284 & 19284 & 15351 & 3933 \\
\hline $\mathrm{GJ} / \mathbf{h})$ & 32.28 & 32.28 & -93.21 & -0.82 & -94.02 & 132.00 & -226.30 & 97.97 & -208.22 & -115.97 & -138.03 & -77.52 & -11.43 & -66.09 & -11.57 & -0.01 & -8.58 & -3.00 & -0.05 & -8.63 & -0.09 & -8.76 & -8.75 & -6.90 & -1.84 \\
\hline emp & 28 & 28 & 28 & 28 & 28 & 28 & 29 & 120 & 190 & 140 & 28 & 140 & 140 & 140 & 40 & 28 & 44 & 44 & 28 & 44 & 28 & 39 & 50 & 50 & 50 \\
\hline ress & 1.00 & 1.00 & 1.00 & 1.00 & 1.00 & 1.00 & 1.00 & 1.00 & 13.72 & 1.00 & 1.00 & 1.00 & 1.00 & 1.00 & 1.00 & 1.00 & 1.00 & 1.00 & 1.00 & 1.00 & 1.00 & 1.00 & 1.00 & 1.00 & 1.00 \\
\hline rea & T-01 & T-02 & PT-03 & T-04 & PT-05 & T-06 & PT-07 & T-08 & PT-09 & T-10 & PT-11 & T-12 & PT-13 & PT-14 & PT-15 & PT-16 & PT-17 & PT-18 & PT-19 & PT-20 & PT-21 & PT-22 & T-23 & PT-24 & PT-25 \\
\hline Wate & 0.189 & 0.189 & 1.000 & .020 & 0.983 & 0.189 & 0.413 & 0.413 & 0.401 & 0.850 & 0.850 & 0.037 & 0.110 & 0.020 & 0.110 & 0.000 & 0.059 & 0.497 & 0.020 & 0.059 & 0.000 & 0.059 & 0.059 & 0.059 & 0.058 \\
\hline igni & 0.119 & 0.119 & 0.000 & 0.000 & 0.000 & 0.119 & 0.085 & 0.085 & 0.085 & 0.000 & 0.000 & 0.155 & 0.023 & 0.186 & 0.023 & 0.000 & 0.026 & 0.000 & 0.000 & 0.026 & 0.000 & 0.025 & 0.025 & 0.025 & 0.025 \\
\hline Cellul & 0.417 & 0.417 & 0.000 & 0.000 & 0.000 & 0.417 & 0.299 & 0.299 & 0.278 & 0.000 & 0.000 & 0.504 & 0.000 & 0.622 & 0.000 & 0.000 & 0.000 & 0.000 & 0.000 & 0.000 & 0.000 & 0.000 & 0.000 & 0.000 & .000 \\
\hline Hemi & 0.129 & 0.129 & .000 & .000 & .000 & 0.129 & 0.093 & 0.093 & 0.005 & 0.000 & 0.00 & 0.008 & 0.000 & 0.010 & 0.000 & 0.000 & 0.000 & 0.000 & .000 & 0.000 & .000 & 0.000 & 0.000 & 0.000 & .000 \\
\hline Ash & 047 & 047 & 000 & 000 & 0.000 & 0.047 & 0.033 & 0.033 & 0.033 & 0.000 & 0.000 & 0.061 & 0.178 & 0.033 & 0.178 & 0.000 & 0.200 & 0.000 & 0.000 & 0.200 & 0.000 & 0.199 & 0.199 & 0.200 & 0.195 \\
\hline Xylos & 000 & 000 & .000 & 000 & .000 & 0.000 & 0.000 & 0.000 & 0.095 & 0.000 & 0.000 & 0.171 & 0.503 & 0.093 & 0.503 & 0.000 & 0.567 & 0.000 & 0.000 & 0.565 & 0.000 & 0.562 & 0.562 & 0.565 & 0.551 \\
\hline than & $0.00 c$ & 00 & .000 & 000 & .000 & .000 & 0.000 & 0.000 & 0.00 & 0.000 & 0.000 & 0.000 & .000 & 0.000 & .000 & 0.000 & 0.000 & .000 & 1.000 & 0.000 & .000 & 0.000 & .000 & 0.000 & .000 \\
\hline $\mathbf{N H}_{3}$ & 000 & 00 & .00 & 000 & .000 & 0.000 & 0.000 & 0.000 & 0.000 & 0.000 & 0.00 & 0.000 & 1.000 & 0.000 & .000 & 1.000 & 0.000 & 0.015 & 0.000 & 0.000 & 1.000 & 0.000 & 0.000 & 0.000 & .00 \\
\hline Gluc & & & .000 & 0 & .000 & 000 & 0.000 & 0.000 & 0.02 & .000 & 0.000 & .042 & 123 & 0.023 & 123 & 0.000 & .139 & .000 & .000 & .139 & 000 & 138 & 38 & 0.139 & 1.13 \\
\hline Furfu & 0 & 0 & 0.000 & 000 & 00 & 000 & 00 & 0.000 & .00 & .006 & $0.0 c$ & 1 & 004 & 01 & 004 & 0.000 & 4 & 0 & .000 & 04 & .000 & 0.004 & 04 & 0.004 & 0.004 \\
\hline $\mathrm{H}_{2} \mathrm{SO}_{4}$ & 0.000 & 0.000 & 1000 & 0.980 & .017 & 0.000 & 0.005 & 0.005 & 0.00 & 0.000 & 0.000 & 1.009 & 0.025 & 0.005 & 0.025 & 0.000 & 0.000 & 0.218 & 0.980 & 0.003 & 0.000 & 0.000 & 0.000 & 0.000 & 0.00 \\
\hline Gypst & 0.000 & 0.000 & 1000 & 0.000 & .000 & 0.000 & 0.000 & 0.000 & 0.000 & .000 & 0.000 & .000 & .000 & 0.000 & .000 & 0.000 & 0.000 & 0.000 & .000 & 0.000 & .000 & 0.005 & .005 & 0.000 & .024 \\
\hline Ca (0 & 0 & 000 & 00 & 000 & 000 & 000 & 0.000 & 0.000 & 0.000 & 0.000 & .00 & 0 & 1.000 & .000 & 000 & 0.000 & 0.000 & 00 & .000 & 00 & .000 & 0.003 & 03 & 0.003 & 03 \\
\hline Aceti & 0.000 & 000 & 0.000 & 000 & 0.000 & 000 & 0.000 & 0.000 & 0.071 & 144 & 0.144 & 12 & 035 & .007 & 035 & 0.000 & .00 & 70 & 0.000 & 0.005 & .000 & 0.005 & 05 & 0.005 & .005 \\
\hline Lactic & 000 & 000 & 000 & 000 & .000 & 000 & .000 & 000 & .00 & 000 & 0.000 & 000 & .000 & .000 & 000 & 0.000 & 0.000 & 0.000 & 0.000 & 0.000 & 0.000 & 0.000 & 0.000 & 0.000 & .000 \\
\hline Cellul & 0.000 & 0.000 & .000 & 0.000 & .000 & .000 & 0.000 & 0 & 0.000 & 0 & 0.000 & 0 & .000 & .000 & 000 & 0.000 & 0.000 & 00 & .000 & 0.000 & 000 & 00 & 00 & 0.000 & 00 \\
\hline Acet & 0.099 & 0.099 & 00 & 000 & 00 & 0.099 & 0 & 0.071 & 0 & 00 & 0 & 000 & 0.000 & 0.000 & 0.000 & 0.000 & 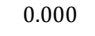 & 00 & 00 & 00 & 000 & .000 & 00 & 0.000 & 0.000 \\
\hline HMF & 0.000 & 0 & 000 & 000 & 000 & 000 & 0. & 000 & & 00 & 00 & & 000 & 000 & 00 & 30 & 0.000 & 00 & 000 & .000 & .000 & 00 & 00 & 0.000 & .000 \\
\hline Levu & 000 & & 000 & & & & & & & & & & & & & & 0.0 & 00 & 0.000 & 0.000 & .000 & & 00 & 0.000 & .000 \\
\hline ormi & 000 & 000 & .000 & 000 & .000 & 0.000 & 0.000 & 0.000 & 0.000 & 0.000 & 0.000 & 0.000 & 0.000 & 0.000 & 0.000 & 0.000 & 0.000 & 0.000 & 0.000 & 0.000 & 0.000 & 0.000 & 0.000 & 0.000 & 0.000 \\
\hline
\end{tabular}


Table S2. Mass and Energy balance for Acid Dehydration Unit

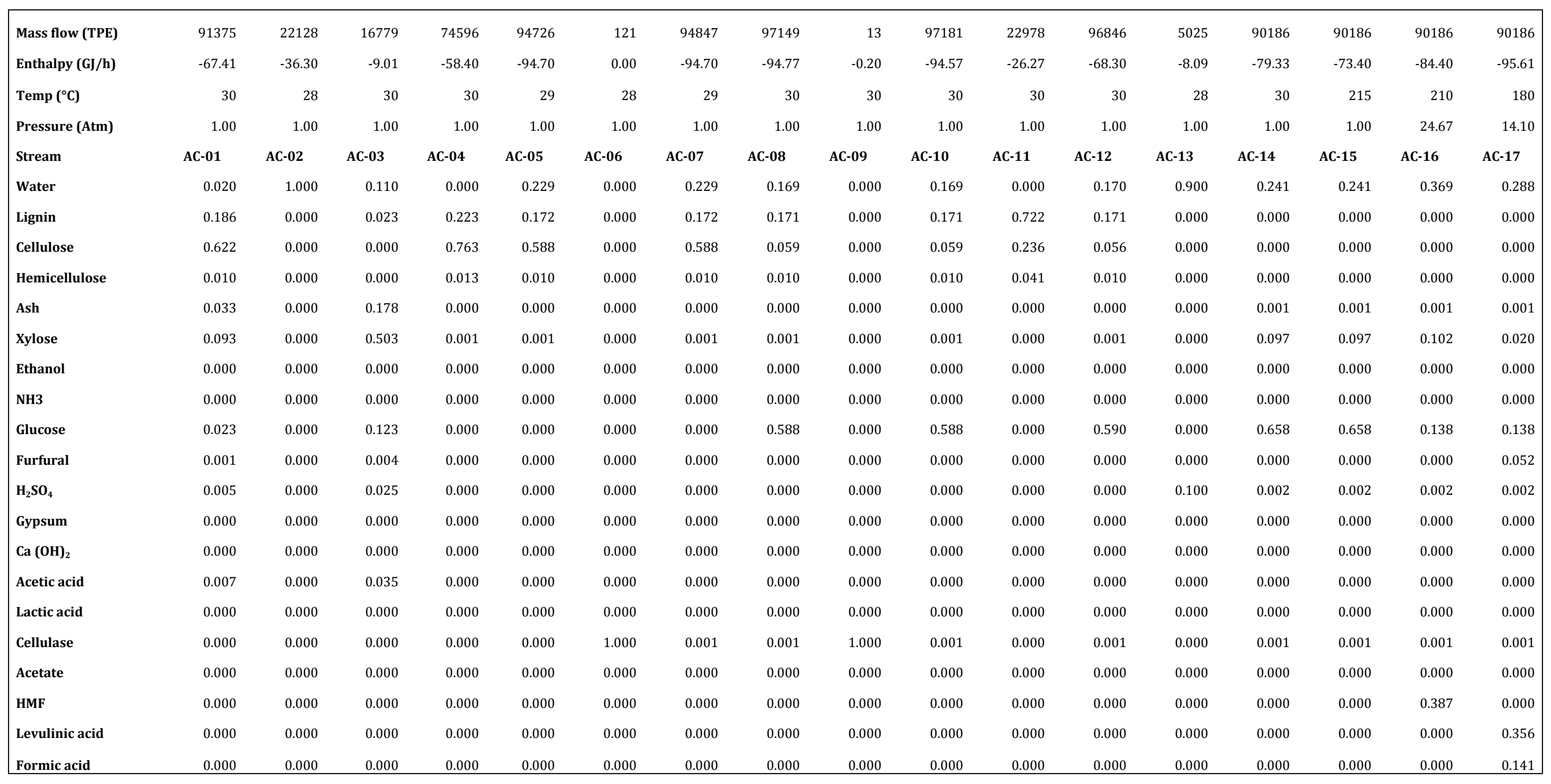


Table S3. Mass and Energy balance for Distillation Unit

\begin{tabular}{|c|c|c|c|c|c|c|c|c|c|}
\hline Mass flow (TPE) & 90186 & 50054 & 40131 & 50054 & 1643 & 1643 & 38488 & 38488 & 38488 \\
\hline Enthalpy flow (GJ/h) & -21.44 & -55.38 & -22.54 & -64.60 & -0.62 & -0.76 & -21.44 & -23.63 & -23.63 \\
\hline Temp $\left({ }^{\circ} \mathrm{C}\right)$ & 30 & 100 & 218 & 28 & 185.1 & 28 & 258 & 28 & 28 \\
\hline Pressure (Atm) & 1.00 & 1.00 & 1.00 & 1.00 & 1.00 & 1.00 & 1.00 & 1.00 & 1.00 \\
\hline Stream & DT-01 & DT-02 & DT-03 & DT-04 & DT- 05 & DT-06 & DT-07 & DT-08 & DT-09 \\
\hline Water & 0.2877 & 0.6128 & 0.0026 & 0.6128 & 0.0627 & 0.0627 & 0.0000 & 0.0000 & 0.0000 \\
\hline Lignin & 0.0001 & 0.0000 & 0.0002 & 0.0000 & 0.0000 & 0.0000 & 0.0002 & 0.0002 & 0.0002 \\
\hline Cellulose & 0.0000 & 0.0000 & 0.0000 & 0.0000 & 0.0000 & 0.0000 & 0.0000 & 0.0000 & 0.0000 \\
\hline Hemicellulose & 0.0000 & 0.0000 & 0.0000 & 0.0000 & 0.0000 & 0.0000 & 0.0000 & 0.0000 & 0.0000 \\
\hline Ash & 0.0006 & 0.0000 & 0.0015 & 0.0000 & 0.0000 & 0.0000 & 0.0016 & 0.0016 & 0.0016 \\
\hline Xylose & 0.0204 & 0.0000 & 0.0005 & 0.0000 & 0.0000 & 0.0000 & 0.0006 & 0.0006 & 0.0006 \\
\hline Ethanol & 0.0000 & 0.0000 & 0.0000 & 0.0000 & 0.0000 & 0.0000 & 0.0000 & 0.0000 & 0.0000 \\
\hline NH3 & 0.0000 & 0.0000 & 0.0000 & 0.0000 & 0.0000 & 0.0000 & 0.0000 & 0.0000 & 0.0000 \\
\hline Glucose & 0.1383 & 0.0000 & 0.0037 & 0.0000 & 0.0000 & 0.0000 & 0.0038 & 0.0038 & 0.0038 \\
\hline Furfural & 0.0523 & 0.0904 & 0.0266 & 0.0904 & 0.6453 & 0.6453 & 0.0002 & 0.0002 & 0.0002 \\
\hline $\mathrm{H}_{2} \mathrm{SO}_{4}$ & 0.0018 & 0.0000 & 0.0049 & 0.0000 & 0.0000 & 0.0000 & 0.0051 & 0.0051 & 0.0051 \\
\hline Gypsum & 0.0000 & 0.0000 & 0.0000 & 0.0000 & 0.0000 & 0.0000 & 0.0000 & 0.0000 & 0.0000 \\
\hline $\mathrm{Ca}(\mathrm{OH})_{2}$ & 0.0000 & 0.0000 & 0.0000 & 0.0000 & 0.0000 & 0.0000 & 0.0000 & 0.0000 & 0.0000 \\
\hline Acetic acid & 0.0001 & 0.0000 & 0.0001 & 0.0000 & 0.0023 & 0.0023 & 0.0000 & 0.0000 & 0.0000 \\
\hline Lactic acid & 0.0000 & 0.0000 & 0.0000 & 0.0000 & 0.0000 & 0.0000 & 0.0000 & 0.0000 & 0.0000 \\
\hline Cellulase & 0.0011 & 0.0000 & 0.0030 & 0.0000 & 0.0000 & 0.0000 & 0.0031 & 0.0031 & 0.0031 \\
\hline Acetate & 0.0000 & 0.0000 & 0.0000 & 0.0000 & 0.0000 & 0.0000 & 0.0000 & 0.0000 & 0.0000 \\
\hline HMF & 0.0000 & 0.0000 & 0.0000 & 0.0000 & 0.0000 & 0.0000 & 0.0000 & 0.0000 & 0.0000 \\
\hline Levulinic acid & 0.3564 & 0.0000 & 0.9503 & 0.0000 & 0.1289 & 0.1289 & 0.9853 & 0.9853 & 0.9853 \\
\hline Formic acid & 0.1413 & 0.2967 & 0.0066 & 0.2967 & 0.1607 & 0.1607 & 0.0000 & 0.0000 & 0.0000 \\
\hline
\end{tabular}

